\title{
Potencial empleo del heptapéptido ATWLPPR como agente de imagen molecular del angiogénesis tumoral
}

\section{Potential use of the ATWLPPR heptapeptide as a molecular imaging agent of tumor angiogenesis}

doi) http:// dx.doi.org/10.35954/SM2019.38.1.4

\author{
Ximena Camacho a (D) https://orcid.org/0000-0002-0755-3834 \\ Carolina Perroni a (D) https://orcid.org/0000-0002-6790-4851 \\ María Fernanda García a (1) https://orcid.org/0000-0002-2918-5761 \\ Juan Pablo Gambini ${ }^{b}$ (1) https://orcid.org/0000-0001-5368-3464 \\ Marcelo Fernández a (1) https://orcid.org/0000-0002-5036-1459 \\ Pablo Cabral a (D) https://orcid.org/0000-0001-7344-2027
}

(a) Departamento de Radiofarmacia. Centro de Investigaciones Nucleares. Facultad de Ciencias. Universidad de la República. Montevideo. Uruguay.

(b) Centro de Medicina Nuclear. Hospital de Clínicas. Facultad de Medicina. Universidad de la República. Montevideo. Uruguay.

\section{RESUMEN}

La Imagenología Molecular comprende la visualización, caracterización y medida de procesos biológicos a nivel molecular y celular en seres humanos $\mathrm{u}$ otros seres vivos. Esta disciplina comprende la realización de imágenes en 2 o 3 dimensiones y su cuantificación en el tiempo. Las técnicas empleadas incluyen, entre otras, a la medicina nuclear. Esto ha llevado a la definición de agentes de imagen moleculares, como sondas empleadas para visualizar, caracterizar y medir procesos biológicos en sistemas vivos, siendo posible emplear moléculas endógenas y exógenas.

En el presente trabajo planteamos desarrollar y optimizar la marcación con el radionucleido emisor gamma, el 99m-Tecnecio, del heptapéptido anti-angiogénico, el ATWLPPR, a modo de evaluar su potencial empleo como agente de imagen molecular de los procesos angiogénicos asociados a cáncer de mama.

Para esto, tanto HYNIC-ATWLPPR como HYNIC -GSG-ATWLPPR fueron adquiridos comercialmente. La marcación con ${ }^{99 \mathrm{~m} T c}$ fue realizada a $50^{\circ} \mathrm{C}$ en presencia de diferentes co-ligandos incluyendo Tricina, Ácido etilendiaminodiacético, Tricina/Ácido etilendiaminodiacético y Tricina/Ácido Nicotínico. Las condiciones de marcación $(\mathrm{pH}$, concentración de co-ligandos, concentración de agente reductor (cloruro de estaño), temperatura y tiempo de reacción) fueron optimizadas en orden, para estandarizar el procedimiento. Se evaluaron las purezas radioquímicas por HPLC. Tanto los coeficientes de partición ( Log $P$ ) y la estabilidad in vitro fueron determinadas a modo de obtener un agente de imagen estable y de alta pureza radioquímica. Se realizaron estudios biológicos in vitro e in vivo para evaluar; a modo de obtener una aproximación al comportamiento biológico de los nuevos radiotrazadores. Logramos radiomarcar los conjugados HYNIC-ATWLPPR y HYNIC-GSG-ATWLPPR con 99m-Tecnecio, obteniendo altas actividades específicas y purezas radioquímicas, al emplear como co-ligandos tanto Tricina como la mezcla Tricina/Ácido Nicotínico. Se lograron obtener complejos estables y con baja lipofilicidad (Log $\mathrm{P}$ de $-3,21 \pm 0,60$ y $-2,70 \pm 0,28$ para 
99mTc-HYNIC-ATWLPPR y de $-2,71 \pm 0,12$ y $-2,97 \pm$ 0,11 para ${ }^{99 \mathrm{~m} T C-H Y N I C-G S G-A T W L P P R}$, empleando Tricina y Tricina/Ácido Nicotínico, respectivamente). El conjugado [99mTc]-HYNIC-GSG-ATWLPPR/ Tricina/Ácido Nicotínico fue el que reveló la mayor afinidad de unión específica por la Neuropilina-1 expresados por la línea celular cáncer de mama asociado a un perfil de biodistribución óptimo para ser empleado como potencial agente de imagen diagnóstico del proceso angiogénico tumoral asociado al cáncer de mama.

PALABRAS CLAVE: ATWLPPR, HYNIC; Imagenología Molecular Oncológica; Neoplasias de la Mama; Tecnecio Tc 99m Sestamibi.

\section{ABSTRACT}

Molecular Imaging comprises the visualization, characterization and measurement of biological processes at the molecular and cellular level in humans or other living beings. This discipline includes 2 or 3 dimension imaging and their quantification over time. Techniques used include, among others, nuclear medicine. This has led to the definition of molecular imaging agents, as probes used to visualize, characterize and measure biological processes in living systems, where endogenous and exogenous molecules can be used.

In the present paper we propose to develop and optimize the gamma emitting radionuclide labeling, $99 \mathrm{~m}$-Technetium, of the anti-angiogenic heptapeptide, the ATWLPPR, in order to assess its potential use as a molecular imaging agent of the angiogenic processes associated with breast cancer.

For this, both HYNIC-ATWLPPR and HYNIC-GSGATWLPPR were commercially purchased. The 99mTc labeling was performed at $50{ }^{\circ} \mathrm{C}$ in the presence of different co-ligands including Tricine, Ethylenediamine diacetic acid, Tricine/Ethylenediamine diacetic acid and Tricine/Nicotinic acid. Labeling conditions ( $\mathrm{pH}, \mathrm{co}-$ ligands concentration of reducing agent (tin chloride), temperature and reaction time) were optimized in order to standardize the procedure.

Radiochemical purities were assessed by HPLC.
Both the partition coefficients $(\log P)$ and in vitro stability were determined in order to obtain a stable imaging agent of high radiochemical purity. Biological studies were carried out in vitro and in vivo to assess; in order to obtain an approximation to the biological behavior of the new radiotracers.

We were able to radiolabel the conjugates HYNIC-ATWLPPR and HYNIC-GSG-ATWLPPR with 99m-Technetium, obtaining high specific activities and radiochemical purities, using as co-ligands both Tricine and the Tricine / Nicotinic Acid mixture. We obtained stable complexes with low lipophilicity (Log $P$ of $-3.21 \pm 0.60$ and $-2.70 \pm 0.28$ for $99 \mathrm{mTc}-\mathrm{HYNIC}$ ATWLPPR and $-2.71 \pm 0.12$ and $-2.97 \pm 0.11$ for 99mTc-HYNIC-GSG-ATWLPPR, using Tricine and Tricine / Nicotinic Acid, respectively).

The conjugate [99mTc]-HYNIC-GSG-ATWLPPR / Tricine / Nicotinic Acid was the one that revealed the highest specific binding affinity for Neuropilin-1 expressed by the breast cancer cell line associated with an optimal biodistribution profile to be used as potential diagnostic imaging agent of the angiogenic tumor process associated with breast cancer.

KEY WORDS: ATWLPPR; HYNIC; Oncological Molecular Imaging; Breast Neoplasms; Technetium Tc 99m Sestamibi.

\section{INTRODUCCIÓN}

La Imagenología Molecular (IM) comprende la visualización, caracterización y cuantificación de procesos biológicos a nivel molecular/celular en seres vivos (1-4), combinando técnicas de imagen mínimamente invasivas, con herramientas de biología molecular/ celular/medicina, etc. $(2,5)$. Es un campo multidisciplinario, dónde las imágenes producidas reflejan in-vivo procesos celulares, permitiendo comprender diversas patologías para un mejor diagnóstico y/o tratamiento (3). La IM juega un rol importante en la evaluación del cáncer, ya que no solo permite visualizar su localización, sino también permite visualizar la expresión y actividad de moléculas específicas (por ejemplo proteasas, protein kinasas) y procesos 
biológicos (por ejemplo apoptosis, angiogénesis, metástasis) que influencian al tumor o su respuesta a la terapia. Se espera que esta información tenga un gran impacto en la detección del cáncer, planificación de un tratamiento individualizado y en el desarrollo de nuevas drogas; así como en nuestra comprensión de los mecanismos subyacentes al origen del cáncer (6). Cabe destacar que en nuestro país se registra un perfil de incidencia del cáncer similar al de países desarrollados, siendo el cáncer de mama el de mayor prevalencia en mujeres.

Debemos tomar en cuenta, que actualmente en cáncer de mama metastásico, no existe un tratamiento para su cura. Un diagnóstico temprano seguido de rápida remoción quirúrgica proporciona la mejor oportunidad de sobrevida. La mamografía no es capaz de detectar metástasis a distancia y por ello técnicas como SPECT/CT y PET/CT son atractivas para lograr una correcta estadificación de los pacientes (7-11). Actualmente, el empleo de 18-FDG para PET, no está indicado para el diagnóstico de cáncer de mama primario (8-11). Sin embargo, a pesar de que el ${ }^{99 m \mathrm{Tc}}$ ([t $\left.1 / 2=6,04 \mathrm{~h}, \mathrm{EY}=140 \mathrm{KeV}(89 \%)\right]$ es el radionucleido más empleado en medicina nuclear por su bajo costo y fácil accesibilidad (12), no existen trazadores específicos para cáncer de mama radiomarcados con ${ }^{99 m} \mathrm{Tc}$. Su desarrollo permitiría que más centros puedan contar los mismos, logrando realizar una mejor estadificación de los pacientes y llevando adelante una medicina personalizada.

La angiogénesis tumoral, definida como el desarrollo de nuevos vasos sanguíneos a partir de los pre-existentes, es esencial para el crecimiento y desarrollo tumoral (13-17). Es controlado por factores pro-lanti-angiogénicos (18), en especial el Factor de Crecimiento Endotelial Vascular (VEGF), que es capaz de unirse a tres receptores (VEGFR-1/-2 y-3) y a dos co-receptores, la Neuropilina-1 y -2 (NRP-1 y -2) localizados en la superficie de las células endoteliales vasculares; conduciendo a la neovascularización del tejido neoplásico (13,17-20). La NRP-1, es capaz de incrementar la unión VEGF/VEGFR-2 hasta 10 veces (15). Es capaz de promover la vas- cularización de tumores prostáticos y es sobreexpresada en otros tipos tumorales como cerebro, mama, colon y pulmón (17,21-36).

El proceso angiogénico es un factor de mal pronóstico (27) y por ello se han desarrollado diversos tratamientos anti-angiogénicos. El primer inhibidor anti-angiogénico desarrollado fue el TNP-470 (28) y actualmente existen más de 300 (29). Uno de estos inhibidores es el ATWLPPR, cuyo blanco es la NRP-1 (30-33), representando así un potencial candidato para la búsqueda de nuevos agentes de imagen oncológicos que sean específicos para la evaluación del proceso angiogénico y progresión tumoral.

En el presente trabajo se pretende desarrollar un potencial agente de imagen basado en la marcación del ATWLPPR con ${ }^{99 m} \mathrm{Tc}$ para diagnóstico del proceso angiogénico tumoral asociado al cáncer de mama. La química del marcado, con ${ }^{99 \mathrm{~m}} \mathrm{Tc}$ de forma directa o a través de agentes quelantes bifuncionales está bien establecida $(34,35)$ y ha sido de amplio uso en nuestro departamento, donde disponemos de una plataforma ya establecida para la generación de este tipo de agentes de imagen (36-40). Se sabe que la secuencia tetrapeptídica del extremo C-terminal del ATWLPPR, -LPPR (-Leu-ProPro-Arg) posee un motivo estructural importante para el reconocimiento del VEGF; donde ambos residuos -Pro (Prolina) son responsables de la determinación estructural, los de -Leu (Leucina) y -Pro son esenciales para el reconocimiento y el de -Arg (Arginina) es fundamental para su actividad biológica. Por lo cual, los cuatro residuos -LPPR son esenciales para la actividad del ATWLPPR (30-33). Por lo tanto, la conjugación con el agente quelante bifuncional se producirá a nivel del extremo N-terminal o vía el linker GSG (Gly-Ser-Gly).

\section{MATERIALES Y MÉTODOS}

\section{1- Síntesis de los péptidos HYNIC-ATWLPPR y HYNIC-GSG-ATWLPPR}

Los heptapéptidos HYNIC-ATWLPPR y HYNIC -GSG-ATWLPPR fueron adquiridos en su forma 
A
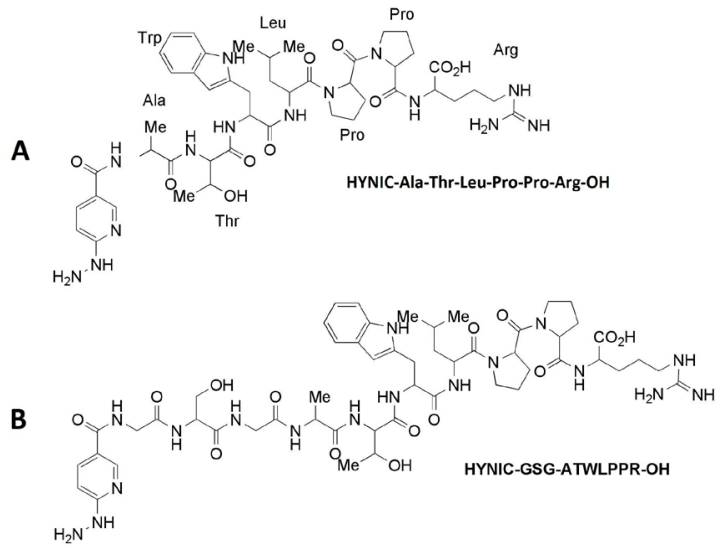

Figura 1. Esquemas de los heptápéptidos HYNIC-

ATWLPPR (A) y HYNIC-GSG-ATWLPPR (B) sintetizados por las empresas Genscript y Siquimia S.A. (Uruguay).

ya derivatizada con el agente quelante bifuncional Succidinimyl-hidrazinonicotinamida (HYNIC) (agente quelante sintetizado en nuestro laboratorio por la Dra. María Fernanda García (40) con una pureza $>95 \%$ (determinada por HPLC-UV). HYNIC -ATWLPPR fue adquirido en forma liofilizada con una pureza del $97,3 \%$ y con un peso molecular 975 $\mathrm{g} / \mathrm{mol}$ (mientras que el PM del ATWLPPR es próxima a $840 \mathrm{~g} / \mathrm{mol}$ ) (figura $1 \mathrm{~A}$ ), de la empresa Genscript USA Inc. HYNIC-GSG-ATWLPPR fue adquirido en su forma ya derivatizada con el agente quelante bifuncional HYNIC (figura 1 B) en forma liofilizada con una pureza del 95\%; de la empresa Siquimia S.R.L.

\section{2- Marcación del péptido con ${ }^{99 m} \mathrm{Tc}$}

Se estudiaron distintas condiciones de marcación con ${ }^{99 m} \mathrm{Tc}$ empleando como co-ligandos los compuestos Tricina, ácido etilenodiamina-N-N'-diacético (EDDA), Tricina/EDDA y Tricina/Ácido Nicotínico (AN), y como agente reductor el cloruro de estaño $\left(\mathrm{SnCl}_{2}\right)$. Todos los solventes empleados fueron previamente purgados con corriente de gas $\mathrm{N}_{2}$.

Las purezas radioquímica ( $\mathrm{PRQ}$ ) de todos los complejos fueron analizados mediante el empleo de un equipo Agilent Serie Infinity 1200, equipado con un detector $G A B$ I Star y utilizando una columna de fase reversa C18 (250 mm x 4,6 x $10 \mu \mathrm{m}$, Restek Ultra), el detector UV se estableció en $280 \mathrm{~nm}$. El método utilizado para la determinación de la $P R Q$ fue el si- guiente: gradiente de $\mathbf{2 0}$ min utilizando como fase móvil Agua/Ácido Trifluoroacético (TFA) 0,1\% (A) y Acetonitrilo (ACN)/TFA 0,1\% (B), 0-5 min 0-45\% B, 5-10 min 45-65\% B, 10-20 min 100\% B.

Para llevar a cabo las respectivas marcaciones se utilizaron $20 \mu \mathrm{g}$ tanto de HYNIC-ATWLPPR como de HYNIC-GSG-ATWLPPR $(1 \mu \mathrm{g} / \mu \mathrm{L})$ almacenado a $-20^{\circ} \mathrm{C}$; a los cuales se les adicionaron:

- $150 \mu \mathrm{L}$ de una solución de Tricina $(100 \mathrm{mg} / \mathrm{mL}$ en $\mathrm{H}_{2} \mathrm{O}$ destilada); $15 \mu \mathrm{L}$ de una solución recién preparada de $\mathrm{SnCl}_{2}(1 \mathrm{mg} / \mathrm{mL}$ en $0.1 \mathrm{M} \mathrm{HCl})$ y 185 -

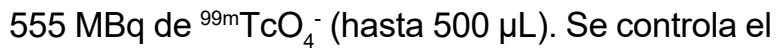
$\mathrm{pH}$ (4.5) y se incuba durante $30 \mathrm{~min}$ a $50^{\circ} \mathrm{C}$ en un baño de agua termostatizado.

- $150 \mu \mathrm{L}$ de una solución de Tricina $(100 \mathrm{mg} / \mathrm{mL}$ en $\mathrm{H}_{2} \mathrm{O}$ ) y $100 \mu \mathrm{L}$ Ácido Nicotínico (AN; $20 \mathrm{mg} / \mathrm{mL}$ en $\mathrm{H}_{2} \mathrm{O}$ destilada); $5 \mu \mathrm{L}$ de una solución de $\mathrm{SnCl}_{2}$ recién preparada $(1 \mathrm{mg} / \mathrm{mL}$ en $0,1 \mathrm{M} \mathrm{HCl}$ ) y $185-555$ $\mathrm{MBq}{ }^{99 \mathrm{~m}} \mathrm{TcO}_{4}^{-}$(hasta $500 \mu \mathrm{L}$ ). Se controla el pH (5) y se incuba durante $30 \mathrm{~min}$ a $50^{\circ} \mathrm{C}$ en un baño de agua termostatizado.

- $150 \mu \mathrm{L}$ de una solución de Tricina $(100 \mathrm{mg} / \mathrm{mL}$ en $\mathrm{H}_{2} \mathrm{O}$ destilada) y $80 \mu \mathrm{L}$ de EDDA ( $20 \mathrm{mg} / \mathrm{mL}$ en $\mathrm{H}_{2} \mathrm{O}$ destilada); $15 \mu \mathrm{L}$ de una solución de $\mathrm{SnCl}_{2}$ recién preparada $(1 \mathrm{mg} / \mathrm{mL}$ en $0,1 \mathrm{M} \mathrm{HCl}$ ) y 185 $555 \mathrm{MBq}{ }^{99 \mathrm{~m}} \mathrm{TcO}_{4}^{-}$(hasta $500 \mu \mathrm{L}$ ). Se controla el $\mathrm{pH}(5)$ y se incuba durante $30 \mathrm{~min}$ a $70^{\circ} \mathrm{C}$ en un baño de agua termostatizado.

- $100 \mu \mathrm{L}$ de EDDA (40 mg/mL en $\mathrm{H}_{2} \mathrm{O}$ destilada); $15 \mu \mathrm{L}$ de una solución de $\mathrm{SnC}_{12}$ recién preparada ( $1 \mathrm{mg} / \mathrm{mL}$ en $0,1 \mathrm{M} \mathrm{HCl}$ ) y $185-555 \mathrm{MBq}^{2}{ }^{99 \mathrm{~m}} \mathrm{TcO}_{4}^{-}$ (hasta $500 \mu \mathrm{L}$ ). Se controla el pH final (5) y se incuba durante 30 min a $70^{\circ} \mathrm{C}$ en un baño de agua termostatizado.

\section{3- Purificación de 99m Tc-HYNIC-ATWLPPR y de 99m Tc-HYNIC-GSG-LHRH}

El control y purificación de los complejos peptídicos marcados se realizaron mediante HPLC utilizando el método que fue mencionado anteriormente. Posteriormente se diluyó la fracción purificada con aproximadamente $500 \mu \mathrm{L}$ de PBS $0,1 \mathrm{M} \mathrm{pH} 7,4$ y el $A C N$ se redujo aplicando 
una corriente de $\mathrm{N}_{2}$. Finalmente, se controla $\mathrm{pH}$ y si es necesario se añade PBS $0,1 \mathrm{M} \mathrm{pH} 7,4$.

\section{4- Coeficiente de reparto o Log $P$}

Para evaluar los valores de Log $\mathrm{P}$, se purificaron por HPLC los conjugados de HYNIC-ATWLPPR y HYNIC-GSG-ATWLPPR/coligando marcados con ${ }^{99 m}$ Tc. El disolvente fue eliminado y el los conjugados marcados $(0,74 \mathrm{MBq})$ fueron reconstituidos en PBS ( $\mathrm{pH} 7,4,0,1 \mathrm{M}, 20 \mathrm{~mL}$ ).

Tubos conteniendo $500 \mu \mathrm{L}$ de Octanol y $500 \mu \mathrm{L}$ de los conjugados marcados disueltos en PBS, fueron vigorosamente agitados durante $1 \mathrm{~min} y$ centrifugados a $14.000 \mathrm{rpm}$ durante $10 \mathrm{~min}$. Seis fracciones de $100 \mu \mathrm{L}$ fueron colectadas de ambas fases, para la medición de sus respectivas cuentas en un contador de pozo de Nal. El coeficiente de reparto se obtuvo como el log (cuentas por min en octanol / cuentas en fase acuosa).

\section{5- Estabilidad in vitro}

\section{1- Estabilidad en PBS}

Se estudió la estabilidad in vitro de todos los

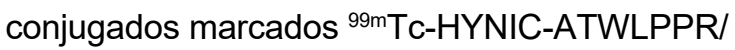
Tricina, ${ }^{99 \mathrm{~m} T c-H Y N I C-G S G-A T W L P P R / T r i c i n a,}{ }^{99 \mathrm{~m} T c}$ -HYNIC-ATWLPPR/Tricina/AN, ${ }^{99 m}$ TC-HYNICGSG -ATWLPPR/Tricina/AN, HYNIC-ATWLPPR/ Tricina/AN, ${ }^{99 m}$ Tc-HYNIC-GSG-ATWLPPR/Tricina/ AN, ${ }^{99 m}$ Tc-HYNIC-ATWLPPR/Tricina-EDDA, ${ }^{99 m} T \mathrm{c}$ -HYNIC-GSG-ATWLPPR/Tricina-EDDA, HYNICATWLPPR/Tricina/AN, ${ }^{99 \mathrm{~m} T c-H Y N I C-G S G-~}$ ATWLPPR/Tricina/AN, ${ }^{99 \mathrm{~m} T c-H Y N I C-~ A T W L P P R / ~}$ EDDA y ${ }^{99 \mathrm{~m} T C-H Y N I C-G S G-A T W L P P R / E D D A . ~}$ Para evaluar la estabilidad, se purificaron por HPLC todos los conjugados radiomarcados por el método previamente descripto. Se incubó una alícuota de $1,85 \mathrm{MBq}$ de cada complejo en $500 \mu \mathrm{L}$ de PBS a $37^{\circ} \mathrm{C}$ hasta 4 hs. Cada experimento fue realizado por triplicado y controlado por HPLC.

\section{2- Estabilidad en Suero Fetal Bovino (SFB)}

Se estudió la estabilidad in vitro de los conjugados marcados empleando los distintos co-ligandos incubando una alícuota de cada complejo peptídico en
SFB a $37^{\circ} \mathrm{C}$, hasta $4 \mathrm{~h}$. Las proteínas fueron precipitadas ACN y centrifugadas $\left(1750 \mathrm{~g}, 5 \mathrm{~min}, 4^{\circ} \mathrm{C}\right)$. Se midió la actividad del precipitado y del sobrenadante en un contador de pozo de Nal. Finalmente, el sobrenadante fue analizado por RP-HPLC para evaluar la estabilidad de los complejos de ${ }^{99 \mathrm{~m} T c}$.

\section{2- Estabilidad en L-Cisteína.}

Se estudió la estabilidad in vitro de los conjugados 99mTc-HYNIC-ATWLPPR/Tricina, ${ }^{99 \mathrm{~m} T c-H Y N I C-G S G}$ -ATWLPPR/Tricina, ${ }^{99 m}$ Tc-HYNIC-ATWLPPR/Tricina/ AN y ${ }^{99 m}$ Tc-HYNIC-GSG-ATWLPPR/Tricina/AN en L-Cisteína mediante el método previamente descripto por Hnatowich et al. (41). Para ello fueron incubados alícuotas de cada complejo radiomarcado disueltos en $500 \mu \mathrm{L}$ de PBS en distintas concentraciones de L-Cisteína $(0,1$ y $1,0 \mathrm{mM})$ a $37^{\circ} \mathrm{C}$, hasta $4 \mathrm{hs}$. Todos los estudios fueron realizados por triplicado y analizadas sus respectivas $P R Q$ fueron evaluados por RP-HPLC.

\section{6- Modelos celulares}

Las líneas celulares humanas de cáncer de mama MDA-MB-231 y de fibroblastos normales NIH3T3, fueron adquiridas de American Type Culture Collection (ATCC). El medio base utilizado para la línea celular MDA-MB-231 fue RPMI-1640 (Capricorn), suplementado con SFB a una concentración final de $10 \%, 2 \mathrm{mM}$ de L-glutamina, $100 \mathrm{U} / \mathrm{mL}$ penicilina y $100 \mu \mathrm{g} / \mathrm{mL}$ streptomicina; mientras el medio base utilizado para la línea celular NIH3T3 fue DMEM alta glucosa (Capricorn), suplementada con SFB a una concentración final de $10 \%, 100 \mathrm{U} / \mathrm{mL}$ penicilina y $100 \mu \mathrm{g} / \mathrm{mL}$ streptomicina y $100 \mu \mathrm{g} / \mathrm{mL}$ de HEPES. Los cultivos fueron incubados a $37{ }^{\circ} \mathrm{C}$ con $5 \%$ de $\mathrm{CO}_{2}$ y una atmósfera del $95 \%$ de humedad.

\section{7- Estudios biológicos in vitro de unión celular}

Se realizaron ensayos de afinidad de unión específica en las líneas celulares mencionadas. Las células fueron cultivadas en medio de cultivo durante dos semanas. Se determinó el número de células y se incubaron $10^{6}$ células en $1,0 \mathrm{ml}$ de medio de cultivo con $\sim 100 \mathrm{nM}$ de 99mTc-HYNIC-ATWLPPR/Tricina/ 
AN y ${ }^{99 m}$ Tc-HYNIC-GSG-ATWLPPR/Tricina/AN por 30,60 y 120 min a $37^{\circ} \mathrm{C}$.

La respectiva unión a la membrana fue determinada mediante un lavado (300 uL) con acetato de sodio (40 mM, pH 4,5) y la internalización fue determinada mediante un segundo lavado (300 uL) de $\mathrm{NaOH} 1 \mathrm{~N}$ por 10 min. La cuantificación de las respectivas cuentas por min (CPM) de los péptidos radiomarcados en cada lavado fue determinado mediante el empleo de un contador gamma automático (Capintec CRC-7, Montvale, N.J., EE.UU.). Cada punto se realiza por cuadruplicado.

Con el fin de confirmar el grado de unión no específica, se llevaron a cabo un ensayo en paralelo incubando las células durante $2 \mathrm{~h}$ con $10 \mu \mathrm{g}$ de los péptidos sin marcar (HYNIC-ATWLPPR y HYNIC-GSG-ATWLPPR) y posteriormente se realizaron las mismas condiciones experimentales anteriormente mencionadas.

\section{8- Estudios de estabilidad biológicos in vivo}

La evaluación biológica in vivo de los complejos ${ }^{99 \mathrm{mTC}} \mathrm{Tc}$ HYNIC-ATWLPPR/Tricina/AN y ${ }^{99 \mathrm{~m} T c-H Y N I C ~-G S G-~}$ ATWLPPR/Tricina/AN se llevaron a cabo mediante la realización de estudios de biodistribución. Todos los procedimientos con animales fueron aprobados por la Comisión Honoraria de Experimentación Animal (Protocolo No: 240011-001280-16). Los ensayos fueron realizados en ratones normales $B A L B / c$, entre 6-10 semanas de edad y 18-24 g. Animales ( $n=5$ por grupo) fueron inyectados por la vena de la cola con aproximadamente 1-3 MBq del complejo de los complejos radiomarcados y sacrificados por dislocación cervical luego de 1 y $2 \mathrm{~h}$. Los tejidos seleccionados (corazón, hígado, pulmones, tiroides, riñones, estómago, bazo, tracto gastrointestinal y vejiga) fueron extirpados, enjuagados de la sangre residual, pesados y sus respectivas radiactividades medidas en un detector $\mathrm{Nal}(\mathrm{TI})$. La sangre y orina fuerontambién colectadosymedidos. La radioactividad en todos los tejidos es expresada como porcentaje de dosis inyectada (\% DI) y como porcentaje de dosis inyectada por gramo de tejido (\% Dl/g).

Los resultados se expresaron como media \pm error estándar. El análisis estadístico se realizó mediante la prueba no apareada t. Fue considerado significativo un valor de $p \leq 0,05$.

\section{RESULTADOS}

\section{1- Marcación con ${ }^{99 m}$ Tc de HYNIC-ATWLPPR y HYNIC-GSG-ATWLPPR}

Primariamente se evaluó por HPLC el perfil de los péptidos sin marcar mediante el empleo del detector UV en las condiciones mencionadas anteriormente, revelando Tiempos de Retención (Tr) de 7,35 y 7,45 min para HYNIC-ATWLPPR (figura 2 A) y HYNIC -GSG-ATWLPPR (figura 2 B).

Posteriormente, se evaluó por HPLC el perfil del ${ }_{99 \mathrm{~m}} \mathrm{TcO}_{4}^{-}$en las condiciones ensayadas, revelando un $\operatorname{Tr}$ de 3,63 min; de lograr establecer su posible presencia en los distintos conjugados como impureza radioquímica (figura 3 ).

Los conjugados HYNIC-ATWLPPR y HYNIC-GSG
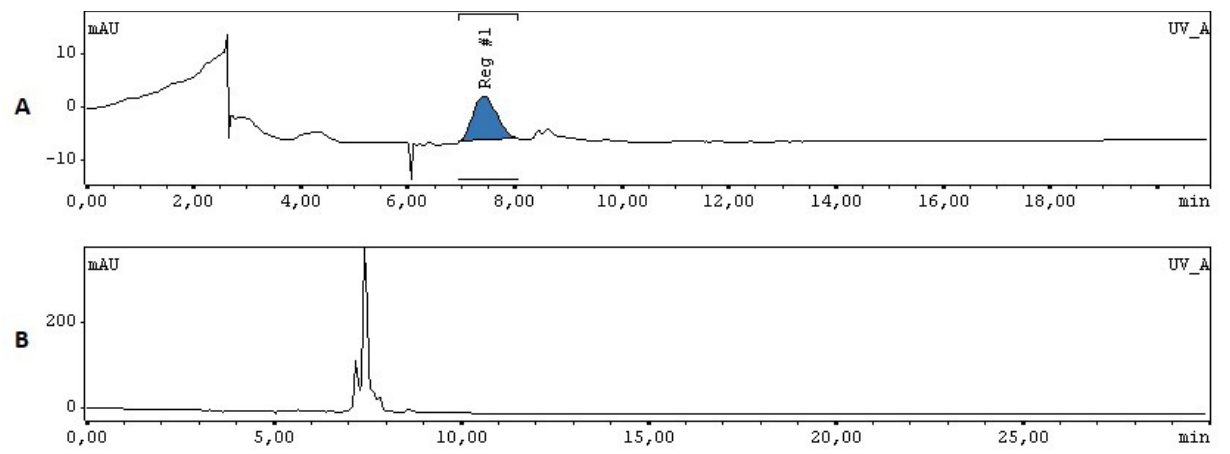

Figura 2. Perfil RT-HPLC de los péptidos HYNIC-ATWLPPR (A) y HYNIC-GSG-ATWLPPR (B) empleando detector UV a $280 \mathrm{~nm}$. Tiempo retención (Tr): 7,35 min y 7,45 min, respectivamente. 


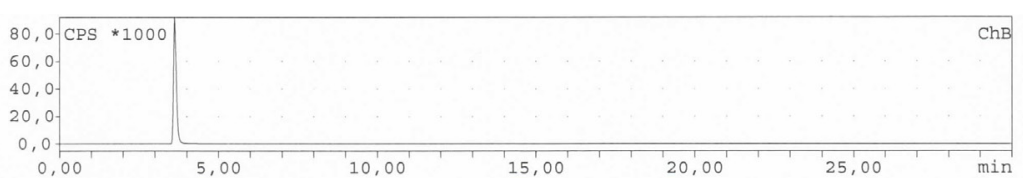

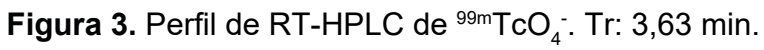
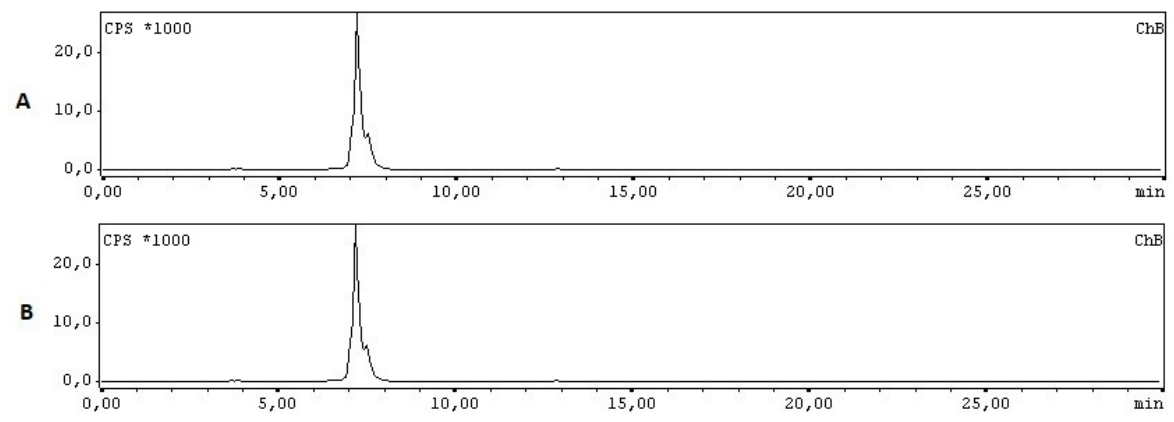

Figura 4. Perfil de RT-HPLC del ${ }^{99 m}$ Tc-HYNIC-ATWLPPR/Tricina (A) y ${ }^{99 m}$ Tc-HYNIC-GSG-ATWLPPR/Tricina (B) en columna C18. Tr: 7,20 min y 7,37 min, respectivamente.
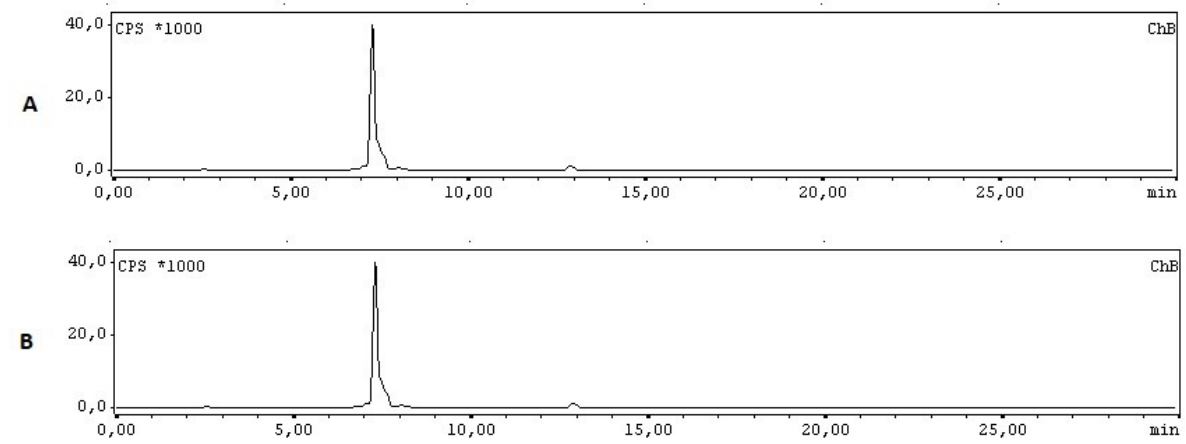

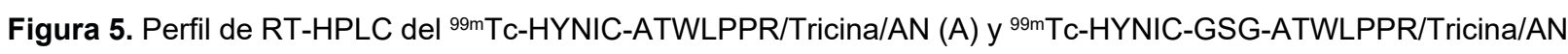
(B) en columna C18. Tr: 7,38 min y 7,23 min, respectivamente.

-ATWLPPR se lograron marcar con rendimientos superiores a $97,6 \pm 3,2 \%$ y $99,5 \pm 2,25 \%$, respectivamente, empleando Tricina como co-ligando a $50{ }^{\circ} \mathrm{C}$ durante $20 \mathrm{~min}$. Ambos complejos se lograron purificar exitosamente a través de HPLC, con Tr de 7,2 y 7,37 min (figura 4 A y B).

Empleando la mezcla de co-ligandos Tricina/AN se obtuvieron rendimientos de marcación de 96,89 \pm $2,50 \%$ y $98,52 \pm 2,51 \%$ para HYNIC-ATWLPPR y HYNIC-GSG-ATWLPPR, respectivamente. Presentando $\operatorname{Tr}$ de 7,38 y 7,23 min (figura 5 A y B).

Empleando la mezcla de co-ligandos Tricina/EDDA se obtuvieron rendimientos de marcación de 84,52 $\pm 4,19 \%$ y $92,68 \pm 5,48 \%$ para HYNIC-ATWLPPR y HYNIC-GSG-ATWLPPR, respectivamente. Presen- tando $\operatorname{Tr}$ de 7,15 y 7,02 min (figura 6 A y B).

Por último, empleando la mezcla el co-ligando EDDA se obtuvieron rendimientos de marcación de 31,93 $\pm 4,19 \%$ y $85,51 \pm 2,41 \%$ para HYNIC-ATWLPPR y HYNIC-GSG-ATWLPPR, respectivamente. Presentando $\operatorname{Tr}$ de 7,40 (figura no mostrada) y 7,23 $\mathrm{min}$ (figura 7).

\section{2- Coeficientes de Reparto o log P}

Se evaluó el carácter hidrofílico de los complejos peptídicos marcados con ${ }^{99 \mathrm{~m} T c}$ utilizando los distintos co-ligandos, mediante el cálculo del coeficiente de reparto octanol-agua. Los valores obtenidos se muestran en la figura 8. En todos los casos el complejo estudiado mostró ser hidrofílico. 

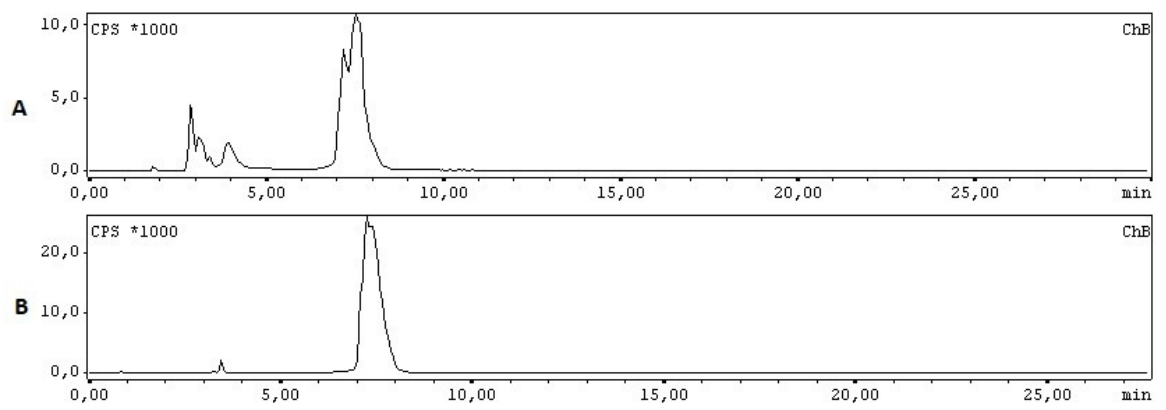

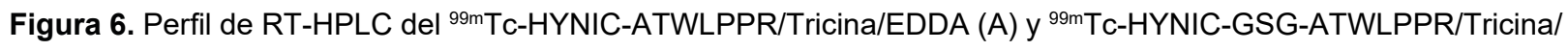
EDDA (B) en columna C18. Tr: 7,15 min y 7,02 min, respectivamente.

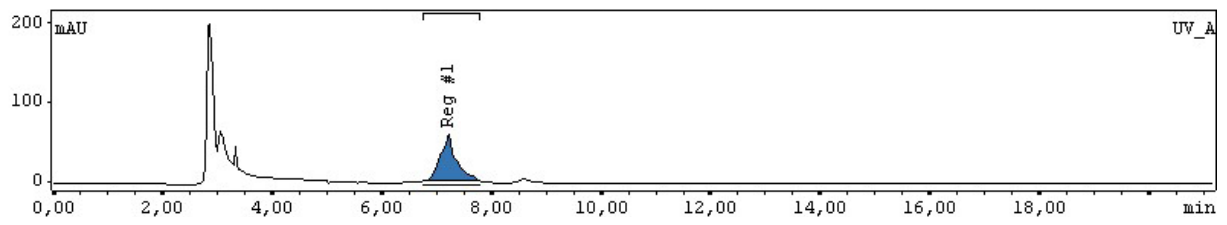

Figura 7. Perfil de RT-HPLC del ${ }^{99 \mathrm{~m} T C-H Y N I C-G S G-A T W L P P R / E D D A ~ e n ~ c o l u m n a ~ C 18 . ~ T r: ~ 7,23 ~ m i n . ~}$

\begin{tabular}{|c|c|c|c|c|}
\hline \multicolumn{5}{|c|}{$\begin{array}{c}\text { Log P para }{ }^{99 m} \text { Tc-HYNIC-ATWLPPR/Tricina; EDDA; Tricina/ } \\
\text { EDDA y Tricina/ AN como co-ligandos }\end{array}$} \\
\hline & Tricina & EDDA & Tricina/EDDA & Tricina/AN \\
\hline $\log P$ & $-3.21 \pm 0.60$ & $-1.35 \pm 0.30$ & $-2.35 \pm 0.15$ & $-2.70 \pm 0.28$ \\
\hline \multicolumn{5}{|c|}{$\begin{array}{c}\text { Log P para }{ }^{99 m} \text { Tc-HYNIC-GSG-ATWLPPR/Tricina; EDDA; Tricina/ } \\
\text { EDDA y Tricina/AN como co-ligandos }\end{array}$} \\
\hline & Tricina & EDDA & Tricina/EDDA & Tricina/AN \\
\hline $\log P$ & $-2.71 \pm 0.12$ & $-1,59 \pm 0.17$ & $-2,77 \pm 0.08$ & $-2.97 \pm 0.11$ \\
\hline
\end{tabular}

Figura 8. Estudio del coeficiente de reparto (Log $P)$ de ${ }^{99 m}$ TC-HYNIC-ATWLPPR/co-ligandos y ${ }^{99 m}$ TC-HYNIC-GSGATWLPPR/co-ligandos.

\section{3- Estabilidad in vitro}

Se estudió la estabilidad in vitro en PBS de los radioconjugados hasta 4 hs. (figura $9 \mathrm{~A}$ y B).

Todos los radiocomplejos resultaron estables, pero sólo los complejos marcados con los co-ligandos Tricina y Tricina/AN presentaron purezas radioquímicas mayores al $90 \%$ hasta las $4 \mathrm{hs}$. del ensayo.

\section{4- Estabilidad en SFB}

También la estabilidad de los radiocomplejos se analizó en SFB hasta 4 hs. (figura 10 A y B). Se observó que al marcar los complejos empleando el co-ligando Tricina y la mezcla de co-ligando Tricina/AN, presentaron una menor unión a proteínas plasmáticas. En especial al emplear la mezcla de co-ligandos Tricina/AN se observó la menor unión

\section{5- Estabilidad en L-Cisteína}

Por último, la estabilidad de los conjugados marcados se evaluó mediante un estudio de transquelación en distintas concentraciones de L-Cisteína (figuras 11 y 12). Se realizó únicamente con los complejos marcado con el co-ligando Tricina y la mezcla de co-ligandos Tricina/AN, debido a que fueron los que presentaron mayor estabilidad tanto en PBS como en SFB.

Se observó que el complejo ${ }^{99 m}$ Tc-HYNIC-ATWLPPR empleando la mezcla de co-ligandos Tricina/AN a 1 $\mathrm{h}$ de incubación presentó la mayor estabilidad, con $P R Q$ de $99,85 \pm 0,30 \%$ y $98,50 \pm 1,85 \%$ en $0,1 \mathrm{mM}$ y $1,0 \mathrm{mM}$ de $\mathrm{L}$ Cisteína, respectivamente. Mientras que, a 4 hs. de incubación, se observaron señales de degradación cercanas a 2 y $5 \%$ en $0,1 \mathrm{mM}$ y $1,0 \mathrm{mM}$ de L Cisteína, respectivamente (figura 11). Mientras que para el complejo ${ }^{99 m}$ Tc-HYNIC-GSGATWLPPR empleando la mezcla de co-ligandos Tricina/AN se observó que a $1 \mathrm{~h}$ de incubación también reveló la mayor estabilidad, con $\mathrm{PRQ}$ de 100,00 \pm $0,50 \%$ y $99,16 \pm 0,84 \%$ en $0,1 \mathrm{mM}$ y $1,0 \mathrm{mM}$ de L-Cisteína, respectivamente. Pero a 4 hs. de incubación, se observaron señales de degradación úni- 


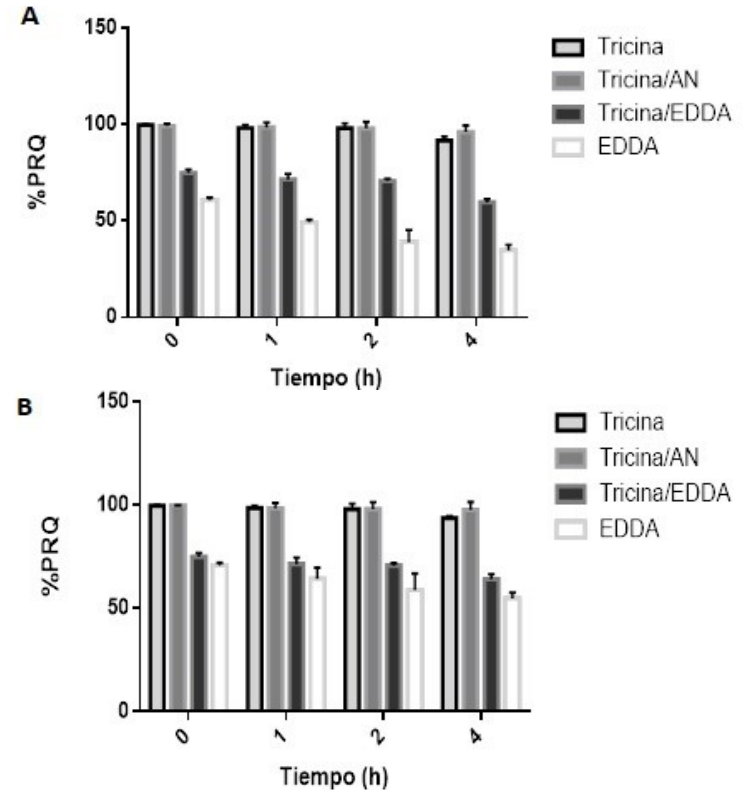

Figura 9. Estudios de estabilidad in vitro en PBS de ${ }^{99 m}$ TC-HYNIC-ATWLPPR (A) y ${ }^{99 m}$ Tc-HYNIC-GSG-ATWLPPR (B). (\% PRQ: \% Pureza radioquímica).

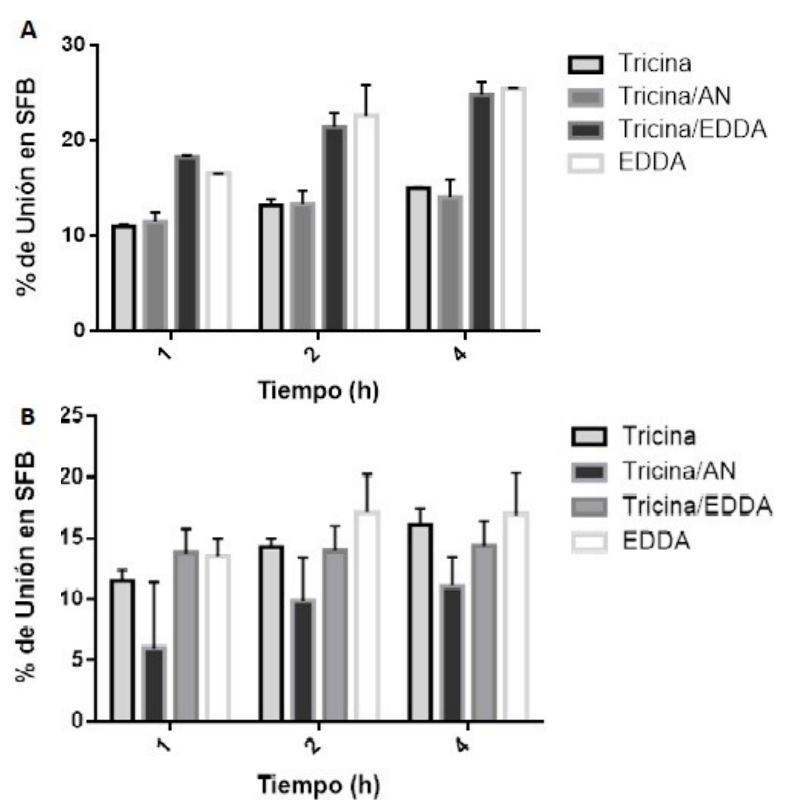

Figura 10. Estudios de estabilidad in vitro en SFB de ${ }^{99 \mathrm{mTC}-}$ HYNIC-ATWLPPR (A) y ${ }^{99 m}$ TC-HYNIC-GSG-ATWLPPR (B).

camente del $1 \%$ a $0,1 \mathrm{mM}$ y $1,0 \mathrm{mM}$ de L-Cisteína, respectivamente (figura 12).

El análisis de la estabilidad en L-Cisteína demostraron que ambos complejos radiomarcados empleando la mezcla de co-ligandos Tricina/AN presentaron las mayores $P R Q$ y estabilidades. Es por lo anterior,

\begin{tabular}{|c|c|c|c|c|c|c|}
\hline \% PRQ & \multicolumn{3}{|c|}{ Tricina } & \multicolumn{3}{c|}{ Tricina/AN } \\
\hline $\begin{array}{c}\text { Tiempo } \\
\text { (H) }\end{array}$ & $\mathbf{1}$ & $\mathbf{2}$ & $\mathbf{4}$ & $\mathbf{1}$ & $\mathbf{2}$ & $\mathbf{4}$ \\
\hline $\begin{array}{c}\text { L-Cisteína } \\
\text { 0.1 } \mathbf{~ m M}\end{array}$ & $\begin{array}{c}96.25 \\
\pm 0.36\end{array}$ & $\begin{array}{c}94.25 \\
\pm 0.75\end{array}$ & $\begin{array}{c}90.28 \\
\pm 1.05\end{array}$ & $\begin{array}{c}99.85 \\
\pm 0.30\end{array}$ & $\begin{array}{c}99.60 \\
\pm 1.25\end{array}$ & $\begin{array}{c}98.55 \\
\pm 1.20\end{array}$ \\
\hline $\begin{array}{c}\text { L-Cisteína } \\
\mathbf{1 . 0} \text { } \mathbf{M M}\end{array}$ & $\begin{array}{c}93.45 \\
\pm 1.25\end{array}$ & $\begin{array}{c}90.18 \\
\pm 0.22\end{array}$ & $\begin{array}{c}88.47 \\
\pm 0.75\end{array}$ & $\begin{array}{c}98.50 \\
\pm 1.85\end{array}$ & $\begin{array}{c}97.20 \\
\pm 1.25\end{array}$ & $\begin{array}{c}94.80 \\
\pm 2.15\end{array}$ \\
\hline
\end{tabular}

Figura 11. Estudio de estabilidad in vitro del complejo ${ }^{99 m}$ Tc-HYNIC-ATWLPPR en distintas concentraciones de L-Cisteína empleando Tricina y la mezcla de co-ligandos Tricina/AN.

\begin{tabular}{|c|c|c|c|c|c|c|}
\hline \% PRQ & \multicolumn{3}{|c|}{ Tricina } & \multicolumn{3}{c|}{ Tricina/AN } \\
\hline $\begin{array}{c}\text { Tiempo } \\
\text { (H) }\end{array}$ & $\mathbf{1}$ & $\mathbf{2}$ & $\mathbf{4}$ & $\mathbf{1}$ & $\mathbf{2}$ & $\mathbf{4}$ \\
\hline $\begin{array}{c}\text { L-Cisteína } \\
\mathbf{0 . 1} \mathbf{~ m M}\end{array}$ & $\begin{array}{c}98.10 \\
\pm 1.24\end{array}$ & $\begin{array}{c}97.52 \\
\pm 1.68\end{array}$ & $\begin{array}{c}96.86 \\
\pm 0.74\end{array}$ & $\begin{array}{c}100.00 \\
\pm 0.50\end{array}$ & $\begin{array}{c}100.00 \\
\pm 2.85\end{array}$ & $\begin{array}{c}99.71 \\
\pm 2.30\end{array}$ \\
\hline $\begin{array}{c}\text { L-Cisteína } \\
\mathbf{1 . 0} \mathbf{~ m M}\end{array}$ & $\begin{array}{c}97.27 \\
\pm 0.85\end{array}$ & $\begin{array}{c}96.18 \\
\pm 1.42\end{array}$ & $\begin{array}{c}93.47 \\
\pm 0.85\end{array}$ & $\begin{array}{c}99.16 \\
\pm 0.84\end{array}$ & $\begin{array}{c}99.05 \\
\pm 2.30\end{array}$ & $\begin{array}{c}98.98 \\
\pm 1.65\end{array}$ \\
\hline
\end{tabular}

Figura 12. Estudio de estabilidad in vitro del complejo ${ }^{99 m}$ Tc-HYNIC-GSG-ATWLPPR en distintas concentraciones de L-Cisteína empleando Tricina y la mezcla de co-ligandos Tricina/AN.

que tanto para los ensayos biológicos in vitro como in vivo, para evaluar los péptidos radiomarcados serán analizados empleando dicha mezcla de co-ligandos.

\section{6- Estudios biológicos in vitro de unión celular}

Para evaluar la afinidad de unión específica de los complejos peptídicos radiomarcados al NRP-1, se realizaron estudios in vitro de unión y de bloqueo mediante la incubación de los respectivos complejos en la línea MDA-MB-231 (cáncer de mama humana). La línea celular NIH3T3 fue empleada como control negativo de unión celular.

En la línea celular MDA-MB-231, empleando 99mTc-HYNIC-ATWLPPR la unión fue de 3,25 \pm $0,64 \%, 5,77 \pm 0,31 \%$ y $8,09 \pm 0,42 \%$; mientras que al emplear ${ }^{99 m}$ Tc-HYNIC-GSG-ATWLPPR la unión fue de 15,42 $\pm 4,04 \%, 17,82 \pm 0,84 \%$ y $21,41 \pm 1,97 \%$ a los 30,60 y 120 min respectivamente. A su vez, se observó que al emplear $20 \mu \mathrm{g}$ de HYNIC-ATWLPPR sin marcar previamente a la adhisión de ${ }^{99 m}$ Tc-HYNIC- ATWLPPR, presentó 

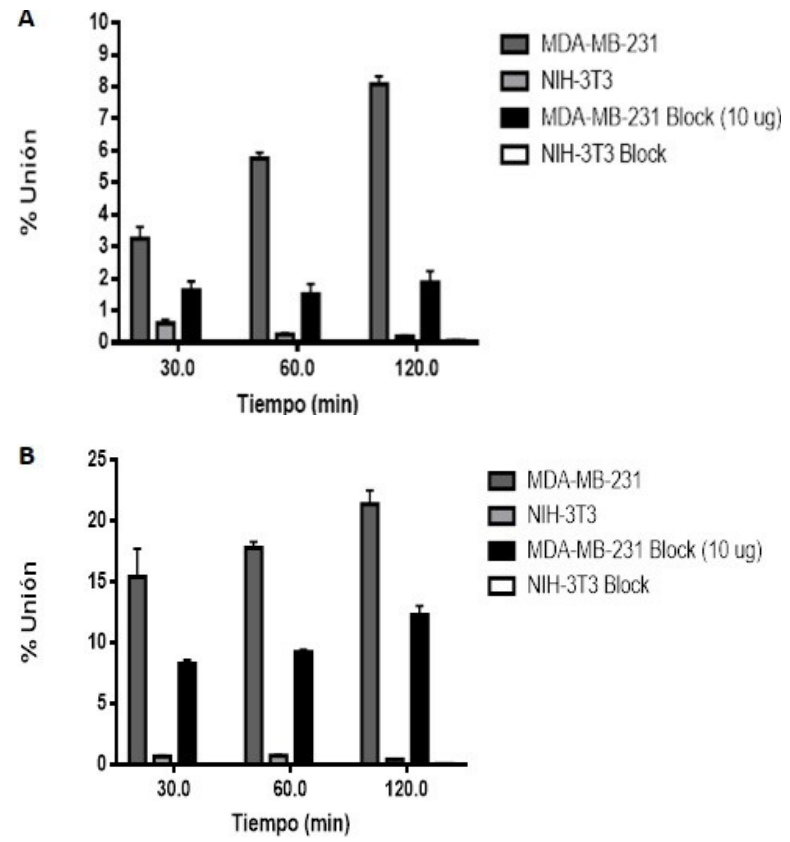

Figura 13. Porcentaje de unión a membrana y bloqueo en las líneas celulares MDA-MB-231 y NIH-3T3 para el complejo ${ }^{99 m}$ Tc-HYNIC-ATWLPPR/Tricina/AN (A) y para ${ }^{99 \mathrm{~m}}$ Tc-HYNIC-GSG-ATWLPPR/Tricina/AN (B).

una inhibición del 45,85\%, 73,66 y 76,76\% a los 30, 60 y 120 min respectivamente (figura $13 \mathrm{~A}$ ); mientras que al emplear HYNIC-GSG-ATWLPPR se observó una inhibición de la adhisión de ${ }^{99 m}$ TcHYNIC-GSG-ATWLPPR de 46,30\%, 48,05\% y 42,55\% (figura $13 \mathrm{~B}$ ).

En la línea celular empleada como control, la NIH-3T3, se observó una unión despreciable de los complejos radiomarcados (figura 13 A y B). Los estudios de unión in vitro llevados a cabo en la línea celular MDA-MB-231 revelaron la efectiva unión de los complejos radiomarcados a la NRP-1 expresada por dicha línea celular.

\section{7- Estabilidad biológica in vivo: estudios de bio- distribución}

La evaluación biológica in vivo de los complejos ${ }^{99 \mathrm{~m} T c-}$ HYNIC-ATWLPPR/Tricina/AN y ${ }^{99 m}$ Tc-HYNIC-GSGATWLPPR/Tricina/AN se llevó a cabo mediante la realización de estudios de biodistribución en ratones Balb/C normales. Las biodistribuciones fueron realizadas luego de 1 y 2 hs. $(n=4)$ (figura 14). La

\begin{tabular}{|c|c|c|c|c|}
\hline & $\begin{array}{c}\text { 99mTc-HYNIC-ATWLPPR/ } \\
\text { Tricina/AN }\end{array}$ & \multicolumn{2}{c|}{$\begin{array}{r}\text { 99mTc-HYNIC- GSG- } \\
\text { ATWLPPR/Tricina/AN }\end{array}$} \\
\hline Tejidos & $1 \mathrm{~h}$ & $2 \mathrm{~h}$ & $1 \mathrm{~h}$ & $2 \mathrm{~h}$ \\
\hline \multicolumn{5}{|c|}{$\%$ Act/g } \\
\hline Sangre & $2.45 \pm 1.29$ & $2.18 \pm 1.10$ & $2.18 \pm 0.96$ & $0.34 \pm 0.11$ \\
\hline Hígado & $1.51 \pm 0.71$ & $1.03 \pm 0.48$ & $0.85 \pm 0.19$ & $0.36 \pm 0.13$ \\
\hline Corazón & $1.97 \pm 1.61$ & $0.79 \pm 0.47$ & $1.55 \pm 0.95$ & $0.89 \pm 0.34$ \\
\hline Pulmón & $1.98 \pm 0.89$ & $1.35 \pm 1.21$ & $1.07 \pm 0.23$ & $0.56 \pm 0.11$ \\
\hline Bazo & $0.97 \pm 0.57$ & $0.43 \pm 0.16$ & $1.92 \pm 0.66$ & $0.48 \pm 0.24$ \\
\hline Riñones & $21.02 \pm 5.29$ & $6.36 \pm 4.22$ & $8.02 \pm 0.99$ & $2.97 \pm 0.14$ \\
\hline Tiroides & $1.48 \pm 1.16$ & $0.68 \pm 0.08$ & $1.73 \pm 0.23$ & $1.56 \pm 0.12$ \\
\hline Músculo & $0.50 \pm 0.28$ & $0.26 \pm 0.06$ & $0.51 \pm 0.03$ & $0.46 \pm 0.36$ \\
\hline Hueso & $0.62 \pm 0.25$ & $0.29 \pm 0.13$ & $1.31 \pm 0.82$ & $0.70 \pm 0.06$ \\
\hline Estómago & $1.09 \pm 0.32$ & $0.49 \pm 0.34$ & $1.92 \pm 0.57$ & $2.34 \pm 0.16$ \\
\hline Intestino & $1.96 \pm 0.83$ & $1.20 \pm 0.23$ & $1.51 \pm 0.36$ & $3.91 \pm 0.49$ \\
\hline \multicolumn{5}{|c|}{$\%$ Act } \\
\hline Intestino & $3.61 \pm 1.77$ & $2.28 \pm 0.62$ & $2.41 \pm 0.67$ & $4.38 \pm 2.97$ \\
\hline Orina & $17.58 \pm 5.29$ & $63.20 \pm 8.79$ & $68.68 \pm 5.81$ & $78.36 \pm 4.95$ \\
\hline
\end{tabular}

Figura 14. Estudio de biodistribución en ratones Balb/C normales a 1 y 2 p.i. de ${ }^{99 m}$ Tc-HYNIC-ATWLPPR/Tricina/ AN y ${ }^{99 m}$ Tc-HYNIC-GSG-ATWLPPR/Tricina/AN. \% Actividad $/$ gramo (\% Act $/ g)(n=4, \%$ Act $/ g \pm D S) ; \%$ Actividad (\% Act) $(n=4, \%$ Act \pm DS).

eliminación de ambos complejos peptídicos resultó ser muy rápida, observándose en vejiga un 17,58 \pm $5,29 \%$ Act y $63,20 \pm 8,79 \%$ Act a 1 y 2 hs. para ${ }^{99 m}$ TcHYNIC-ATWLPR/Tricina/AN y 68,68 $\pm 5,81 \%$ Act y $78,36 \pm 4,95 \%$ Act a 1 y 2 hs. para ${ }^{99 m}$ Tc- HYNICGSG-ATWLPR/Tricina/AN, respectivamente. A su vez se observó una retención inespecífica a nivel renal de ambos complejos. La captación en el resto de órganos normales fue < de $2 \%$ Act $/ g$.

\section{DISCUSIÓN}

Mediante el presente trabajo logramos realizar el estudio preliminar del heptapéptido ATWLPPR como potencial agente imagen diagnóstico de los procesos angiogénicos asociados a cáncer de mama.

Para poder hallar el mejor complejo peptídico para el radiomarcado se evaluó la posibilidad de unir al agente quelante bifuncional de dos formas distintas: la primera, se unió de forma directa el extremo $\mathrm{N}$-terminal del heptapéptido al HYNIC y la segunda, mediante la modificación empleando un linker; el tripéptido, Gly-Ser-Gly (GSG) y posterior conjugación 
con el agente bifuncional HYNIC. Mediante ambas formas se logró radiomarcar ambos conjugados de manera óptima con el radionucleido ${ }^{99 \mathrm{~m}} \mathrm{Tc}$.

Según el empleo del co-ligando se lograron variadas actividades específicas, todas superiores al $80 \%$, excepto al emplear el co-ligando EDDA al radiomarcar el complejo ${ }^{99 m}$ Tc-HYNIC-ATWLPPR/EDDA. Como evaluación pre-clínica, realizamos estudios in vitro de estabilidad, unión a proteínas y lipofilicidad a modo de predecir la posible biodistribución en el organismo.

El estudio de pureza radioquímica de todos los conjugados y su subsiguiente purificación por HPLC permitió detectar la presencia de los conjugados marcados con ${ }^{99 \mathrm{~m}} \mathrm{Tc}$ de forma eficiente. Las mayores purezas radioquímicas se lograron al utilizar los co-ligando Tricina y Tricina/AN; estos también mostraron ser los más hidrofílicos (menor Log $\mathrm{P}$ ) y los que presentan mayor estabilidad, tanto en PBS como en SFB $37^{\circ} \mathrm{C}$ hasta 4 hs.; por lo cual evaluamos la estabilidad de estos complejos hasta $4 \mathrm{hs}$. con distintas concentraciones de L-Cisteína, logrando adecuados rendimientos en especial al emplear el conjugado 99mTc-HYNIC-GSG-ATWLPPR/Tricina/AN, debido a esto fue el seleccionado para llevar a cabo los estudios biológicos in vitro e in vivo.

Los estudios biológicos in vitro en la línea celular de cáncer de mama, así como en la línea celular NIH-3T3 de fibroblastos normales (control negativo) se realizaron para evaluar la afinidad de unión del conjugado ${ }^{99 m}$ TcHYNIC-GSG-ATWLPPR/Tricina/AN a la NRP-1. Se observó que el complejo radiomarcado presentó una significativa unión en la línea de cáncer de mama evaluada (15-20\%) en comparación con la línea control $(<0,5 \%)$.

Se observó a su vez una inhibición $>40 \%$ al emplear el péptido sin marcar, lo que indica la especificidad de la unión del radioconjugado a la NRP-1 expresado por la línea celular ensayada.

Por último se verificó la efectiva estabilidad observada del conjugado ${ }^{99 m}$ TcHYNIC-GSG-ATWLPPR/ Tricina/AN mediante estudios de biodistribución en ratones Balb/C normales, revelando un perfil de bio- distribución muy adecuado para el desarrollo de un agente de imagen diagnóstico, con elevada eliminación renal y baja retención inespecífica a nivel renal (más del $60 \%$ las $1 \mathrm{~h}$ p.i.), la cual nos alentaría a realizar futuras imágenes moleculares en modelos murinos portadores de tumores de mama inducidos.

\section{CONCLUSIONES}

Mediante el presente estudio logramos llevar a cabo la optimización de la marcación con ${ }^{99 m} \mathrm{Tc}$ de los conjugados HYNIC-ATWLPPR y HYNIC-GSG-ATWLPPR de forma fácil, rápida y sencilla. Se encontró que el complejo ${ }^{99 m}$ Tc-HYNIC-GSG-ATWLPPR/Tricina/AN presenta la $>P R Q$ y estabilidad en todos los ensayos realizados, no requiriendo una posterior purificación. ${ }^{99 \mathrm{~m} T c-H Y N I C-G S G-A T W L P P R / T r i c i n a / ~}$ AN reveló una adecuada afinidad de unión por su receptor en modelos in vitro de cáncer de mama, como también se observaron perfiles de eliminación ideales, representando de esta forma un prometedor agente de imagen molecular para el diagnóstico de la expresión de la NRP-1 oncológico. A futuro pretendemos realizar estudios imagenológicos en modelos tumorales de cáncer de mama.

\section{AGRADECIMIENTOS}

Agradecemos a la Agencia Nacional de Investigación e Innovación (ANII, Uruguay), a la Comisión Sectorial de Investigación Científica (CSIC) y a los programas ProlnBio-Medicina y PEDECIBA-Química. No existen conflictos de intereses. 


\section{REFERENCIAS}

(1) Mankoff DA. A definition of molecular imaging. J Nucl Med 2007; 48(6):18N-21N.

(2) Weissleder R, Mahmood U. Molecular Imaging. Radiology 2001; 219:316-33.

(3) Willmann JK, van Bruggen N, Dinkelborg LM, Gambhir SS. Molecular imaging in drug development. Nat Rev Drug Discov 2008; 7(7):591-607.

(4) James ML, Gambhir SS. A molecular imaging primer: modalities, imaging agents, and applications. Physiol Rev 2012; 92:897-965

(5) Nichol C, Kim EE. Molecular imaging and gene therapy. J Nucl Med 2001; 42(9):1368-1374.

(6) Weissleder R. Molecular imaging in cancer. Science 2006; 312(5777):1168-71.

(7) Buist DS, Porter PL, Lehman C, Taplin SH, White E. Factors contributing to mammography failure in women aged 40-49 years. J Natl Inst 2004; 96:1432-1440.

(8) Avril N, Menzel M, Dose J, Schelling M, Weber $W$, Jänicke $F$, et al. Glucose metabolism of breast cancer assessed by 18-FDG PET: histologic and immunohistochemical tissue analysis.

J Nucl Med 2001; 42:9-16.

(9) Avril N, Rosé CA, Schelling M, Dose J, Kuhn $W$, Bense S, etal. Breastimaging with positronemission tomography and fluorine-18 fluorodeoxyglucose: use and limitations. J Clin Oncol 2000; 18:3495-3502.

(10) Isasi CR, Moadel RM, Blaufox MD. A metaanalysis of FDG PET for the evaluation of breast cancer recurrence and metastases.

Breast Cancer Treat 2005; 90:105-12.

(11) Lonneux M, Borbath I, Berlière M, Kirkove C, Pauwels S. The place of whole-body PET FDG for the diagnosis of distant recurrence of breast cancer. Clin Positron Imaging 2000; 3:45-49.

(12) Dilworth JR, Parrott JS. The biomedical chemistry of technetium and rhenium.

Chem Soc Rev 1988; 27:43-55.
(13) Carmeliet P; Jain RK. Angiogenesis in cancer and other diseases. Nature 2000; 407:249-57.

(14) Carmeliet P. Angiogenesis in life, disease and medicine. Nature 2005; 438:932-936.

(15) Ferrara N. Role of vascular endothelial growth factor in physiologic and patholgic angiogenesis: therapeutic implications.

Semin Oncol 2002; 29(6):10-14.

(16) Ferrara N, Gerber HP, LeCouter J. The biology of VEGF and its receptors.

Nat Med 2003; 9(6):669-676.

(17) Dvorak HF. Angiogenesis: update 2005.

Thromb Haemost 2005; 3:1835-42.

(18) Carmeliet P. Angiogenesis in health and disease. Nat Med 2003; 9:653-60.

(19) Dvorak HF, Brown LF, Detmar M, Dvorak AM. Vascular permeability factor/vascular endothelial growth factor and the significance on microvascular hyperpermeability in angiogenesis.

Curr Top Microbiol Inmunol 1999; 237:97-132.

(20) Partanen TA, Paavonen K. Lymphatic versus blood vascular endothelial growth factors and receptors in humans. Microsc Res Tech 2001; 5:108-121.

(21) Ellis LM. The role of neuropilins in cancer. Mol Cancer Ther 2006; 5(5):1099-107.

(22) Lampropoulou A, Ruhrberg C. Neuropilin regulation of angiogenesis.

Biochem Soci Trans 2014; 42(6):1623-28.

(23) Miao HQ, Lee P, Lin H, Soker S, Klagsbrun M. Neuropilin-1 expression by tumor cells promotes tumor angiogenesis and progression.

FASEB Journal 2000; 14(15):2532-39.

(24) Fakhari M, Pullirsch D, Abraham D, Paya K, Hofbauer R, Holzfeind P, etal. Selective upregulation of vascular endothelial growth factors receptors neuropilin-1 and -2 in human neuroblastoma.

Cancer 2002; 94(1):258-63. 
(25) Bachelder RE, Crago A, Chung J, Wendt MA, Shaw LM, Robinson G, et al. Vascular endothelial growth factor is an autocrine survival factor for neuropilin-expressing breast carcinoma cells. Cancer Res 2001; 61(15):5736-40.

(26) Barr MP, Byrne AM, Duffy AM, Condron $\mathrm{CM}$, Devocelle $\mathrm{M}$, Harriott $\mathrm{P}$, et al. A peptide corresponding to the neuropilin-1-biniding site on VEGF(165) induces apoptosis of neuropilin-1expressing breast tumor cells.

British J Cancer 2005; 92(2):328-33.

(27) Tas F, Duranyildiz D, Oguz H, Camlica H, Yasasever V, Topuz E. Circulating serum levels of angiogenic factors and vascular endohtelial growth factors receptors 1 and 2 in melanoma patients. Melanoma Res 2006; 16(5):405-11.

(28) Ingber D, Fujita T, Kishimoto S, Sudo K, Kanamaru $\mathrm{T}, \mathrm{Brem} \mathrm{H}$, et al. Synthetic analogues of fumagilin that inhibit angiogenesis and supress tumour growth. Nature 1990; 348:555-7

(29) Hurwitz H, Fehrenbacher L, Novotny W, Cartwright T, Hainsworth J, Heim W, et al. Bevacizumab plus irinotecan, fluorouracil, and leucovorin for metastatic colorectal cancer.

N Engl J Med 2004; 350:2335-42.

(30) Binétruy-Tournaire R, Demangel C, Malavaud B, Vassy R, Rouyre S, Kraemer M, et al. Identification of a peptide blocking vascular endothelial growth factor (VEGF)-mediated angiogenesis. EMBO J 2000; 19(7):1525-33.

(31) Perret GY, Starzec A, Hauet N, Vergote J, Le Pecheur M, Vassy $R$, et al. In vitro evaluation and biodistribution of a $99 \mathrm{mTc}$-labelled anti-VEGF peptide targeting neuropilin-1.

Nucl Med Biol 2004; 31:575-81.

(32) Starzec A, Vassy R, Martin A, Lecouvey M, Di Benedetto $M$, Crépin $M$, et al. Antiangiogenic and antitumor activities of peptide inhibiting the vascular endothelial growth factor binding to neuropilin-1. Life Sci 2006; 79:2370-81.
(33) Tirand L, Frochot C, Vanderesse R, Thomas $\mathrm{N}$, Trinquet E, Pinel S, et al. A peptide competing with VEGF165 binding on neuropilin-1 targeting of a chlorine-type photosensitizer and potentiates its photodynamic activity in human endothelial cells. J Control Res 2006; 111:153-64.

(34) Jurisson S, Berning D, Jia W, Ma D. Coordination Compounds in Nuclear Medicine.

Chem Rev 1993; 93(3):1137-56.

(35) Dilworth JR, Parrottb JS. The biomedical chemistry of technetium and rhenium.

Chem Soc Rev 1988; 27:43-55.

(36) Camacho X, Machado CL, García MF, Fernández M, Oddone N, Benech J. Tocilizumab labeling with $99 \mathrm{mTechnetium} \mathrm{via} \mathrm{HYNIC} \mathrm{as} \mathrm{a}$ molecular diagnostic agent for Multiple Myeloma. Anticancer Agents Med Chem 2017; 17(9):1267-1277.

(37) Camacho X, Machado CL, García MF, Gambini JP, Banchero A, Fernández $M$, et al. Technetium$99 \mathrm{~m}$ - or Cy7-Labeled Rituximab as an Imaging Agent for Non-Hodgkin Lymphoma.

Oncology 2017; 15(92):229-42.

(38) Calzada V, García MF, Alonso-Martínez LM, Camacho X, Goicochea E, Fernández $M$, et al. Fab(nimotuzumab)-HYNIC-99mTc: Antibody fragmentation for molecular imaging agents.

Anticancer Agents Med Chem 2016; 16(9):1184-9.

(39) Camacho X, García MF, Calzada V, Fernández M, Alonso O Gambini JP, et al. 99mTc-labeled Bevacizumab vía HYNIC for Imaging of Melanoma. J Anal Oncology 2014; 3:53-64.

(40) García MF, Calzada V, Camacho X, Goicochea E, Gambini JP, Quinn TP, et al. Microwave-assisted Synthesis of HYNIC Protected Analogue for 99mTc Labeled Antibody.

Curr Radiopharm 2014; 7(2):84-90.

(41) Hnatowich J, Virzi F, Fogarasi M, Rusckowski $M$, Winnard $P$ Jr. Can a cysteine challenge assay predict the in vivo behavior of ${ }^{99 \mathrm{mT}} \mathrm{Tc}$-labeled antibodies? Nucl Med Biol 1994; 21(8):1035-44. 